\title{
Wild edible plant use in Tshopo District, Democratic Republic of Congo
}

\author{
Céline Termote \\ Supervisor: Patrick Van Damme \\ Department of Plant Production, Ghent University
}

Public defence: 25 April 2012

Since the late 1980 s and early 1990 , and in conjunction with an increasing global concern about environmental issues, increased attention for rural poverty and the emergence of the concept 'sustainable development', there has been an increasing support from the scientific community, NGOs and policy makers to address issues of biological conservation and poverty reduction as interrelated and not as mutually exclusive goals. Agricultural biodiversity and its potential as a driver of economic growth, food security and natural resource conservation has gathered an increasing amount of interest since the Convention on Biological Diversity was voted in 1992. Even though the nature of the evidence is still circumstantial, it is a reasonable and compelling assumption that increased agricultural and forest biological diversity leads to a more varied diet, which in turn improves specific health outcomes. Nutrition and biodiversity also feature directly in the Millennium Development goals (MDGs), in Goal I, Target 2, 'Halve the proportion of people who suffer from hunger'; and goal 7 , 'Ensure environmental sustainability'.

Despite the country's high endowment with natural resources, $90 \%$ of the 60 million Congolese inhabitants is poor, whereas the majority of them lives in rural areas. $70 \%$ of DRC's inhabitants depend on the forest for their daily livelihoods. Valorising wild edible plants (WEP), and non-timber forest products (NTFP) in general, which constitute a particular niche of the poorest, will thus contribute to poverty alleviation and increased nutrition security in a DRC context. Although the potential of NTFPs to contribute to poverty alleviation is recognized in the country's Poverty Reduction Strategy Papers (DSCRP 2006,2011 ), both documents deplore the huge gap of knowledge in this sector.

Despite their enormous intrinsic biodiversity, the Congolese forests are the least documented in Africa, not only in terms of their potential for industrial timber exploitation, but also in terms of socio-economic and cultural value to the local populations (firewood, medicine, shelter, tools, game, caterpillars, mushrooms, honey, WEPs, dyes, cultural and spiritual values, etc.). In order to underpin political decisions and to find innovative ways for managing DRC's precious natural resources, there is an urgent need to revitalize forestry and agricultural research in the country.

Until now, and notwithstanding the richness in biodiversity and cultures, WEPs have been little studied in Tshopo District, c.q. DRC. Tshopo District has an enormous agricultural potential, but nevertheless food security remains structurally precarious. Challenged with the consequences of food insecurity in their daily activities and aware of the underutilized potential of WEPs, some local NGOs have put forward that there must be opportunities to valorize local wild foods for better nutrition and health. 
The main objective of this $\mathrm{PhD}$ study was to contribute to the valorization of local WEPs for improved food security and livelihoods in Tshopo District, DRC. Therefore, we used a multidisciplinary approach including the domains of ethnobotany, market analysis and nutrition, with WEPs at the centre. As a long-term vision, we see the most interesting WEPs being promoted, domesticated, integrated into farmers' fields and commercialized for I) improved nutrition and health; 2) increased and diversified farmers' income; and 3) sustained cultural well-being without compromising preservation of natural resources. This work presents information on WEP uses, present and future commercialization possibilities, and current and potential contributions of WEPs to local diets, nutrient intake and dietary adequacy. Because further in-depth study of all WEP species documented in this work is almost impossible, we paid, special attention to the prioritization of species. The results of this work can then constitute the basis for further study, participatory domestication and value chain development of priority WEP species, i.e. species with highest nutritional and socio-economic potential, and cultural acceptance.

We started our investigations with a thorough ethnobotanical study to inventory the indigenous knowledge on WEPs in 3 ethnic groups (Turumbu, Mbole and Bali) in 3 different territories of Tshopo District. WEPs were collected in 3 villages per ethnic group to constitute a reference herbarium and their properties recorded during focus group discussions. In total, we documented 166 WEP species and 2 varieties in 71 plant families from which 198 plant parts are used for 228 different specific food uses. Compared to available literature, 72 species reported in our study were cited for the first time as WEP in the region. In addition, 136 of the 166 WEP species were shown to be used for other purposes such as medicines, materials and arts, cultural uses, construction, fuels, bait, fodder or poison. Moreover, we compared inter- and intra-ethnic differences in WEP knowledge and found that only $2 \mathrm{r} \%$ of the species were known and used by all 3 ethnic groups. Further inventorying WEP knowledge in the other ethnic groups of Tshopo District would thus certainly add new species and uses to our WEP list. Besides differences in cultural habits and preferences for WEPs, we observed that the various ethnic groups in Tshopo District are faced with different opportunities and constraints such as market access. Consequently, we should make a distinction between species with overall regional importance and ethnospecific species when it comes to priority setting for further study, participatory domestication and/or conservation.

Preferences in taste and commercial, nutritional and cultural value of both wild fruits and wild vegetables, were discussed during participatory ranking exercises within the Turumbu, Mbole and Bali ethnic groups. Results show that, for all ethnic groups considered together, overall liking was highest for Anonidium mannii, followed by Landolphia owariensis and Tetracarpidium conophorum in the wild fruits category, and Megaphrynium macrostachyum in the wild vegetables category. We did not perform any separate preference ranking exercises neither for spices nor for stimulant nuts. However, the ethnobotanical inventories showed that the wild spices Piper guineense and Scorodophloeus zenkeri together with the stimulant nuts Cola acuminata and Garcinia kola are used in all three ethnic groups for a great number of purposes, whereas they are also highly embedded in traditional 
culture. According to us, they should thus be added to the list of most-preferred species. Subsequently, we analyzed WEP markets in Kisangani in relation to number and socioeconomic characteristics of sellers, WEP species and quantities offered, prices and periodicity in offer. From the 166 WEPs known by the Turumbu, Mbole and Bali ethnic groups in Tshopo District, only 15 WEPs were seen to be sold on Kisangani markets by a small number of 'ad hoc' traders, who often switch easily to other products when they become available. The September-October period and 'Marché Central' are most important for WEP trade in Kisangani. Added values and net incomes generated through WEP sales highly vary, even between traders of a same species. Until now, most WEPs are harvested for autoconsumption. As a consequence WEP markets in the region remain underdeveloped, although, a certain form of organization was found in the Gnetum africanum trade with Kinshasa as main destination. In general, traders mentioned lack of transport and market infrastructure, lack of storage space, (il)legal taxes, lack of credit facilities, insufficiency of WEPs to purchase and low purchasing power of consumers, as the main constraints in WEP trade.
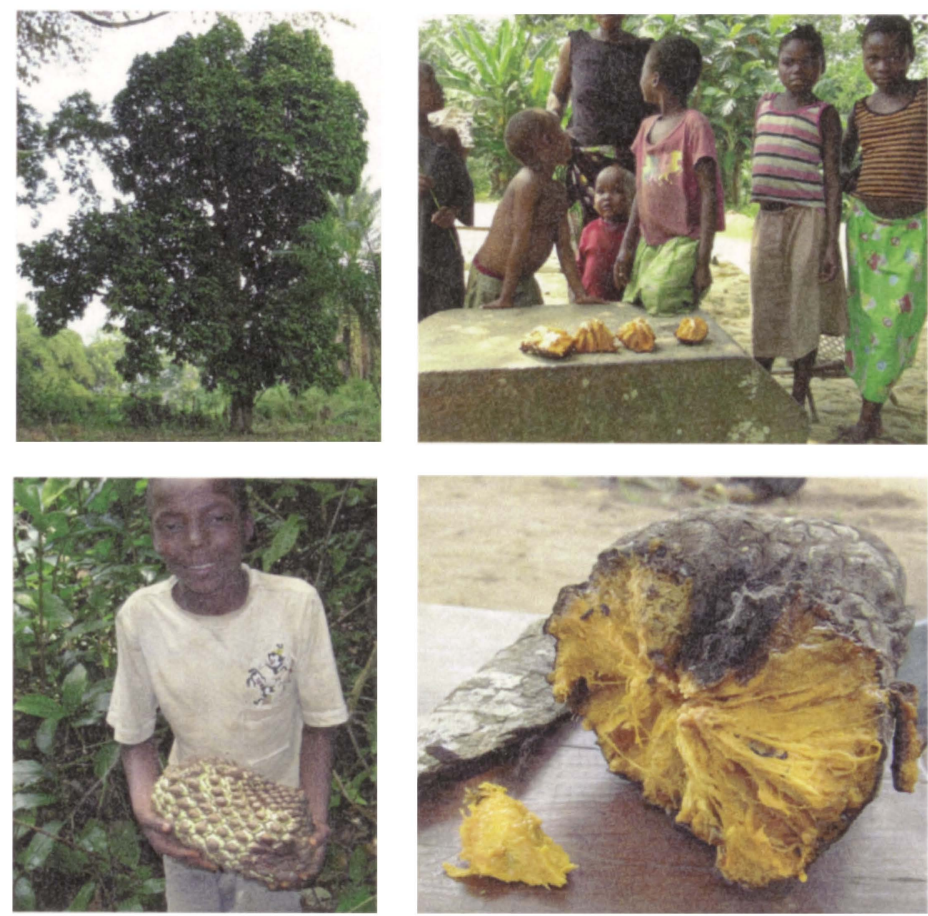

Anonidium mannii tree and fruits; above right : children selling pieces of the fruit along the roadside 

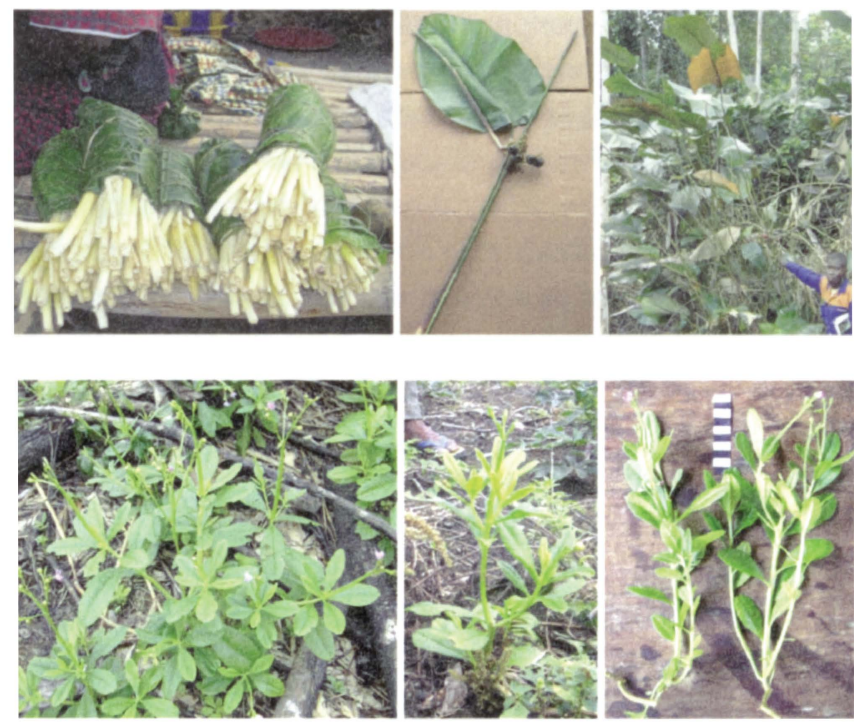

Wild vegetables highly appreciated by the Turumbu; Megaphrynium macrostachyum above) and Talinum triangulare (below)

Based on the nature of species sold as well as on the socio-economic characteristics of our interviewees, we identified four types of traders: subsistence traders, traders diversifying in number of WEPs sold, traders diversifying in number of income activities, and specialized traders. This typology provides valuable insights for further market chain organization and development, especially because selling particular WEPs seems to be linked to particular trader socio-economic characteristics.

International, national as well as local markets exist for Gnetum africanum leaves, Piper guineense fruits, and nuts of Cola acuminata and Garcinia kola. The species which has the highest demand and also the most dynamic traders is undoubtedly G. africanum. However, for long-term sustainability of successful NTFP commercialization, (participatory) domestication and integration of G. africanum into agroforestry systems is urgently needed. Wild spices (P. guineense, C. acuminata and G. kola), on the other hand, are so far only locally sold, mainly in micro-quantities by extremely poor, elderly widows. Organizing the wild spices market chains for more efficiency will require special strategies to protect these elderly (widow) women. Otherwise, the chances are that they will not be able to compete with the more dynamic traders that may enter the market when value chains become more efficient.

From the market study, it further follows that Landolphia owariensis and Tetracarpidium conophorum are priority wild fruits for local market development. They are both easier to transport and less perishable than the very big Anondium mannii fruits, whereas they are also sold by a relatively higher number of traders. Although the latter fruit had the highest score in the preference ranking exercises performed during our ethnobotanical investigations, better transport, processing and/or conservation of A. mannii will be necessary 
for its market to expand. Local market development for wild vegetables will require additional nutritional education and sensitization about the many advantages of diversified diets and wild vegetables for good health in order to stimulate demand side.

To assess the contribution of WEPs to dietary quality, we estimated the usual dietary intake from 2 multiple-pass 24 h dietary recalls of 363 urban (Kisangani) and 129 rural (Yaoseko) women within the period of highest WEP availability. Contrarily to what was expected, only 15 WEPs were found to occur and then only in a marginal number of $24 \mathrm{~h}$-recalls. The most noteworthy contribution came from the semi-wild safou fruit (Dacryodes edulis), reported in $30.1 \%$ of all recalls and contributing $4.8 \%$ of total energy intake in Yaoseko village and this within the safou season (about 3 months per year). We further found that total energy intake of the interviewed women was rather low, with a high percentage energy coming from fats. The micronutrients of major concern were niacin, folate, vitamin B-12, iron, zinc and calcium with more than $75 \%$ of women having intakes below the recommended dietary allowances. Despite the precarious nutrition security, urban as well as rural inhabitants in this highly biodiverse region do not valorize their knowledge on WEPs to complement their diets. Overall consumption of WEPs in the sample was too low to achieve adequate dietary intake or nutrition security. So, it is wrong to assume that biodiverse-rich environments automatically lead to better diets. However, this does nót mean that WEPs and agrobiodiversity, in general, could not contribute to better nutrition security.
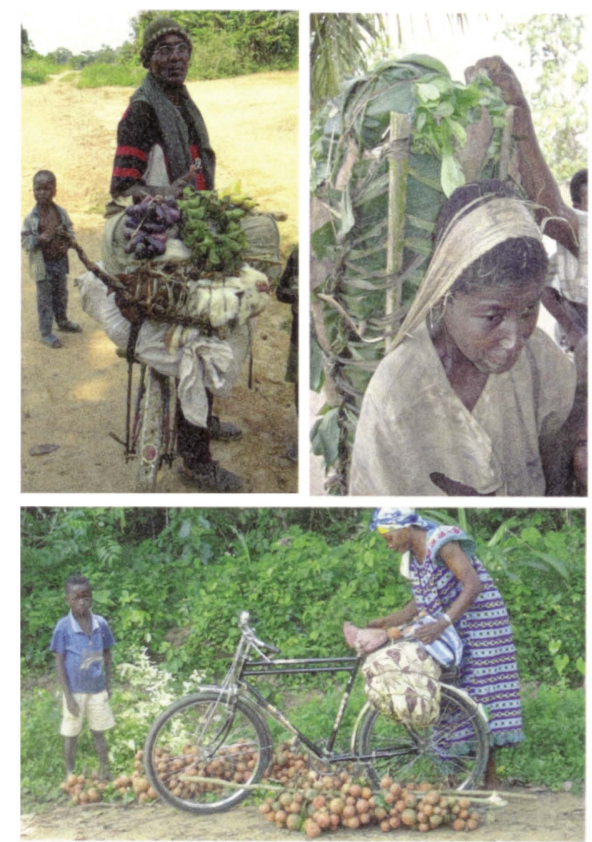

Above left: Man transporting safou (Dacryodes edulis, purple) and tito (Tetracarpidium conophorum, green); above right: woman coming from the field, bringing melelu (Talinum triangulare) for today's meal; below: woman having harvested liyo fruits (Landolphic owariensis) 
It is clear that, in the given situation, promotion of WEPs alone will not solve all underlying causes of nutrition insecurity and poverty in the region, but they can at least contribute more to better local diets and incomes then they currently do. A lot of WEPs with proven nutritional qualities, such as Gnetum africanum and Treculia africana (and others of which the nutritional qualities are not yet known by science), are present in the region. Before getting indefinitely lost, indigenous knowledge on wild edible species and their dietary use need further research to capture the potential of biodiversity and ameliorate diet adequacy.
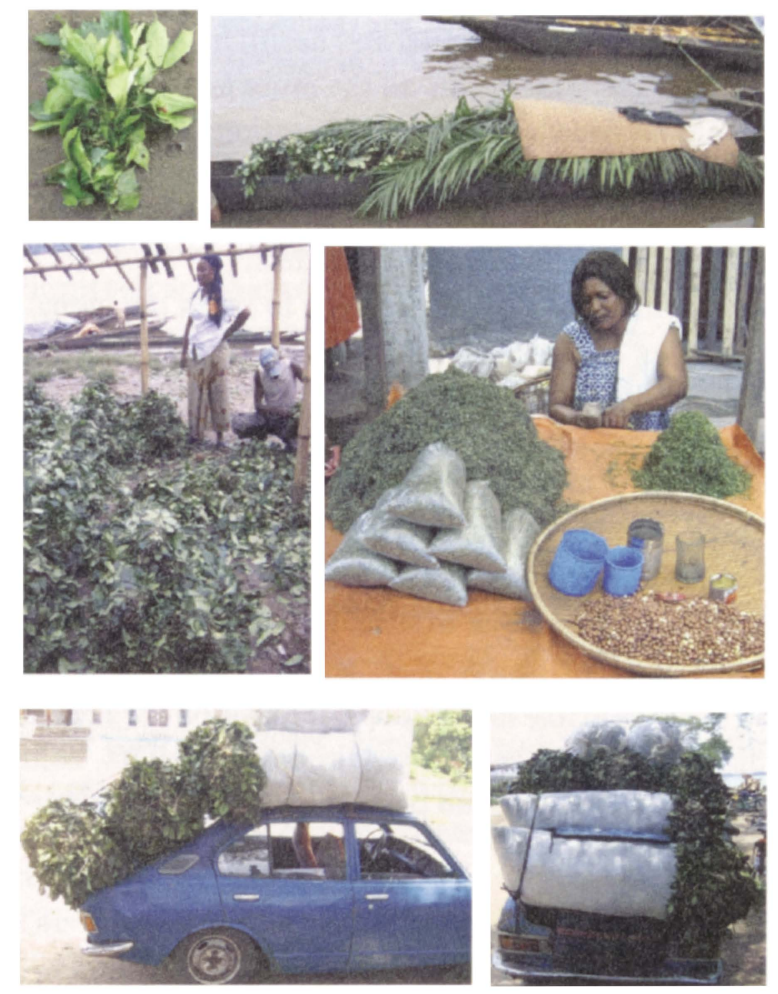

Gnetum africanum trade; above left: bundle of Gnetum africanum; right: canoe filled with Gnetum africanum bundles arriving at IAT market; middle left: fresh Gnetum africanum leaves at IAT market; right: selling dried Gnetum africanum at the Central Market; below: transport of Gnetum africanum to the airflight companies

Research into agrobiodiversity and agroforestry starting from local needs and indigenous knowledge, and backed up by sound scientific research principles will be indispensable to create innovative, resilient agricultural models able to produce healthy foods in a sustainable way while at the same time conserving biodiversity for future generations. In line with this, we could e.g. consider the promotion and integration of indigenous fruits, vegetables, tubers, spices, etc. in home gardens and agroforestry systems. Nonetheless, participatory domestication of indigenous fruit (and medicinal) trees and the integration 
of them into agroforestry systems together with value chain development for these species, belongs to the core activities of ICRAF's work in West and Central Africa. A partnership was established and since 2009 ICRAF extended its activities in Tshopo District. At present they are working with three local farmer groups, one local NGO and the local universities, UNIKIS and IFA-Yangambi.

Even though nowadays WEPs are not widely traded nor used in Tshopo District, the indigenous knowledge on them is still present and culturally well-defined. It should thus be possible to identify, embrace and build upon local socio-cultural values which are likely to enhance WEP consumption and trade. From our (scientific) side, we should urgently generate and make available more nutritional and health information on WEPs to validate the existing local knowledge. This seems all the more necessary because the local population itself - being told for decades that only conventional crops are good for health - is requesting this kind of information and would most benefit from it. Through the recognition and valorization of their own knowledge, local populations can reach a sustainable way of living in conjunction with the modern context.

Undoubtedly, a lot of work remains to be done in order to reach the global aim of improving food security, diversifying and increasing farmers' incomes and protecting the rainforest from overexploitation through participatory domestication and improved market chain organization. However, we hope to have set one step into the right direction by making available information on WEP uses, commercialization and consumption, and by providing insight into how local WEPs can be valorized and prioritized for further research. Further research is needed to map the indigenous knowledge and preferences on WEPs in the other ethnic groups in the region; to study into detail the value chains of the priority WEP species in order to define pro-poor strategies for further development of these chains; to analyze and make available nutritional composition data of the WEPs; to study and conceptualize the many links between biodiversity, nutrition, culture and livelihoods in the region; to evaluate domestication possibilities and sustainable harvest techniques, etc. 